\title{
BACKWARD np SCATTERING WITH A POLARIZED TARGET*
}

\author{
P. R. ROBRISH, O. CHAMBERLAIN, R. D. FIELD Jr., R. Z. FUZESY, W. GORN, C. C. MOREHOUSE \\ T. POWELL, S. ROCK, S. SHANNON, G. SHAPIRO and H. WEISBERG \\ University of Calıforna Lawrence Radiation Laboratory. Berkeley California 94720 USA \\ and
}

\author{
M. J. LONGO \\ University of Michigan Ann Arbor. Michtgan 48104. USA
}

Recerved 16 March 1970

\begin{abstract}
We measured the polarization parameter $P$ in neutron-proton elastic scattering near the backward direction, using a polarized proton target. Measurements covered the range of incident neutron momenta from 1.0 to $55 \mathrm{GeV} / c$ and of four-momentum transfer squared $u$ from -0005 to $-0.5(\mathrm{GeV} / c)^{2}$.
\end{abstract}

Measurements of the differential cross section for np scattering near $180 \mathrm{deg}$, sometimes referred to as np charge-exchange scattering, have shown a very sharp peak near $u=0[1]$. There have been many attempts to explain the behavior of the cross section using Regge models, but only those models employing conspiring trajectories [2] or absorptive corrections [3] seem to have had some measure of success. There has also been some success using modified one-pionexchange models [4]. We therefore decided to measure the polarization in order to provide new information about this reaction and to help to distinguish between the varıous theoretical models.

We measured the polarization parameter by directing a neutron beam having a broad spectrum of momenta from 1 to $5.5 \mathrm{GeV} / c$ at a polarized target. Using a magnetic spectrometer, we determined the direction and momentum of protons emerging near the forward direction. An array of neutron counters was used to measure the direction of the outgoing neutron and, somewhat crudely, its velocity. By using both the velocity of the neutron and the angular correlation between the directions of the outgoing neutron and proton, we were able to determine whether a given event was elastic scattering. We used the measured momentum of the out-

* Work supported by the U.S. Atomic Energy Commision and O.N.R. National Science Foundation Grant GP 9438 . going proton along with its direction to calculate the momentum of the incident neutron. $P$ was determined from the change in counting rate $I$ of scattered particles upon reversal of the target polarization $P_{\mathrm{T}}$ according to

$$
I=I_{0}\left[1+P P_{T} \cdot \frac{\hat{\boldsymbol{k}}_{\text {neutron }}^{\text {lncident }} \times \hat{\boldsymbol{k}}_{\text {neut ron }}^{\text {final }}}{\sin \theta_{\text {neutron }}^{\text {final }}}\right]
$$

The experiment was performed at the Bevatron in a neutron beam produced at 0 deg from a $7.5-\mathrm{cm}$-long $\mathrm{Cu}$ target located in the external proton beam. The defining aperture of the collimator was $1.6 \mathrm{~cm}$ in diameter at $4.6 \mathrm{~m}$ from the production target, and gave a beam spot of about $3 \mathrm{~cm}$ diam at our target, which was $8.5 \mathrm{~m}$ from the production target. Four and one-half radiation lengths of $\mathrm{Pb}$ were put in the beam line $1 \mathrm{~m}$ down-stream of the production target to convert $\gamma$-rays. Sweeping magnets before and after the collimator removed charged particles from the beam. The neutron intensity at our target was about $3 \times 10^{6}$ neutrons per 1011 incident protons.

The polarized target consisted of about $6 \mathrm{~g} / \mathrm{cm}^{2}$ of $\mathrm{La}_{2} \mathrm{Mg}_{3}\left(\mathrm{NO}_{3}\right)_{12} \cdot 24 \mathrm{H}_{2} \mathrm{O}$ in which the free protons in the water of hydration ( $3 \%$ by weight) were polarized by dynamic nuclear orientation. The average polarization during the experiment was $50 \%$. The sign of the polarization was reversed about every 90 minutes. The NMR signal used to obtain the polarization of the target was measured after every Bevatron pulse. 

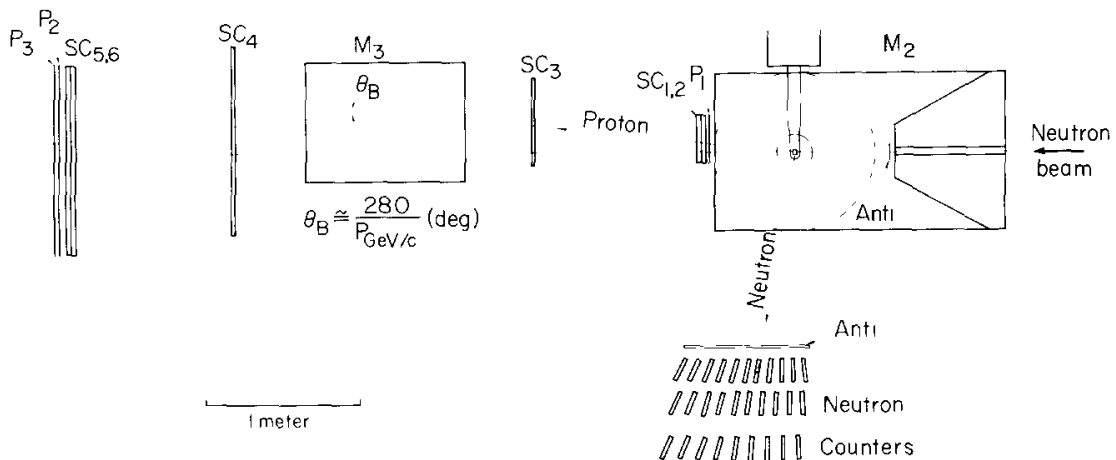

Fig 1 Side view of the experimental arrangement M2 is the polarized target magnet. The neutron bema entered through a hole in the return yoke of this magnet. M3 is the analyzing magnet in the proton spectrometer.

Fig。 1 is a diagram of the apparatus used to detect the final-state particles. Outgoing protons were detected in a magnetic spectrometer consisting of three counters P1, P2, P3, and six wire spark chambers, SC1 SC6, with magnetostrictive readout, three before and three after the spectrometer magnet. This spectrometer was used to measure the momentum, and direction of each proton.

The data from both the wire chambers and the electronics associated with the neutron counters described below were read into a PDP-5 computer and transferred to magnetic tape between Bevatron pulses. The computer also recorded the polarized target information and provided displays which allowed us to monitor the operation of the experiment.

The neutrons were detected in an array of 30 plastic scintillation counters each $2.5 \times 15$ $\times 96 \mathrm{~cm}^{3}$, where the long axis of each counter was perpendicular to the plane of $\mathrm{fig}$. 1 . By measuring the time of flight difference of the scintillation light to two photomultiplier tubes, one located at each end of the counter, we determined the location of the detected neutron vertex along the long axis of each counter to about $\pm 4 \mathrm{~cm}$. We staggered the counters in three rows as shown in fig. 1 in order to minimize the effect of the "cross talk" caused by the scattering of recoil particles into adjancent counters. We identified the neutron interaction vertex with the row closest to the target in which a count registered and demanded that only one counter in that row have fired $(\approx 7 \%$ of the events were lost because of the requirement). The electronic circuitry was arranged so that the time difference information which was recorded always came from this first nonblank row alone; timing information from other rows was lost. For each event we also measured the time difference between counter P1 and a photomultiplier at one end, in order to get time-of-flight information on the finalstate neutron.

Since the experimental data on each event contained the momentum and direction of the recoll proton as well as the time of flight and $\mathrm{d}_{1}-$ rection of the scattered neutron it was quite straightforward to determine which events were elastic scattering on free (polarized) protons. Using the information on the recoil proton, we calculated the kinematic parameters that the finalstate neutron should have had if the event were elastic $n-p$ scattering. We plotted for each event the difference between the observed parameters and those calculated from the proton information. These parameters included the neutron scattering angle $\theta$, the neutron azimuthal angle $\varphi$, and the neutron time of flight. Our resolution was such that in a multidimensional plot there was a clear-peak at the origin due to elastic $n-p$ scattering. The sharpness of the peak, typically $\pm 1^{\circ}$ in $\theta$ and $\pm 2^{\circ}$ in $\varphi$, made it quite evident that the peak could not be ciue to either inelastic $n-p$ scattering or quasielastic scattering in the heavy elements within the target. This was confirmed by a study of those events significantly noncoplanar (deviating in $\varphi$ ) and by observing scattering from a dummy target similar in nuclear composition to the polarized target, but containing no hydrogen。 Typically we obtained a ratio of eleastic peak to background of about $6: 1$.

We determined the shape of the small background lying under the peak both by using the non-coplanar events and by using the data from the dummy target. The background was normalized to events for which $\theta$ was clearly incosistent with elastic $n-p$ scattering. The results of both types of background subtraction were quite consistent. 

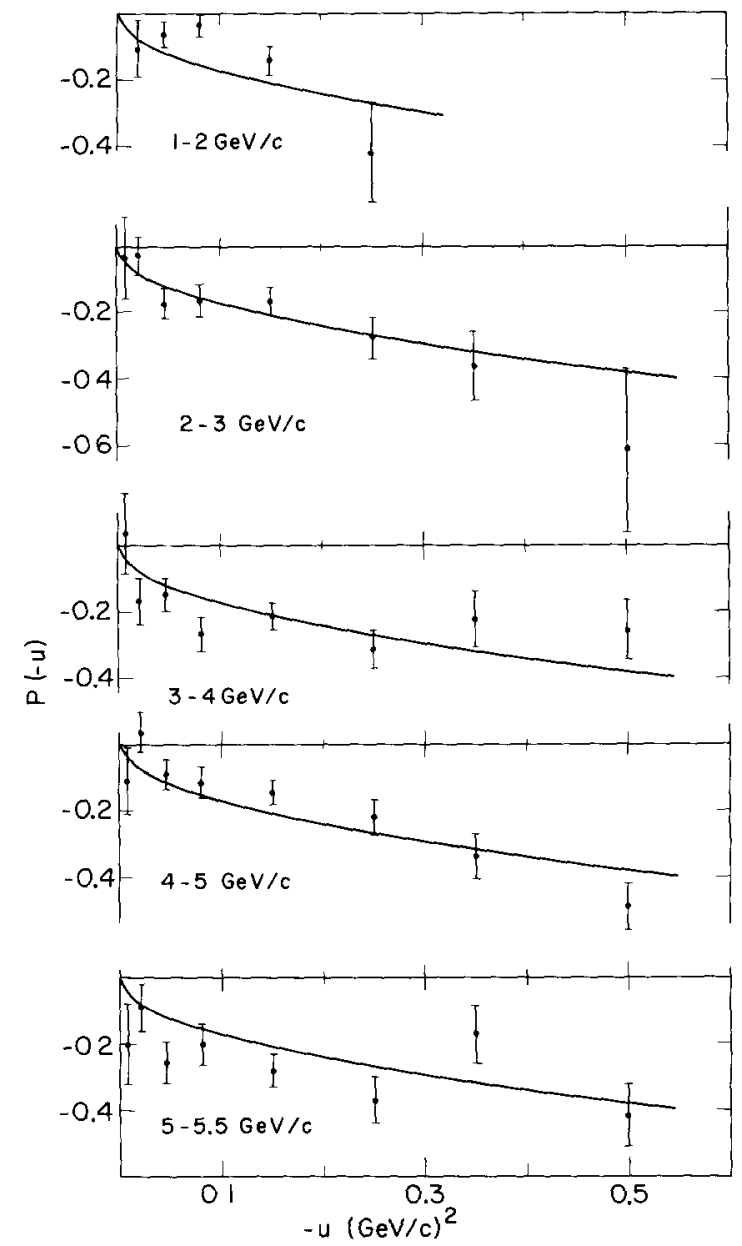

Fig. 2. Measured values of the polarızation $P$ in backward np scattering for the indicated values of incident neutron laboratory momentum. Errors due counting statistics only are shown. There is an additional overall multiplicative factor of $(100 \pm 0.05)$ due to uncertainty in the average target polarization.

The error in our results due to uncertainty in the background subtraction is negligible compared with the error from counting statistics. Checks of the internal consistency of the data indicate that systematic errors, such as instabilities of the monitors and detectors, are also negligible. The only significant error in our data, other than that from counting statistics, is thought to be given by an overall normalization factor of $(1 \pm 0.05)$ corresponding to our est1mate of the uncertainty in the measurement of the target polarization.

The results of our preliminary analysis, which includes about 2000000 event candidates of which about 60000 were elastic events, are shown in fig. 2. The solid lines represent the purely empirical function $P=-0.5 \sqrt{-u} / m_{\mathrm{p}}$, and are presented to show that this function represents our data fairly well. Our sign convention for $P$ corresponds to defining the normal to the scattering plane as the vector product of the incident and scattered neutron momenta. The features of the data which are probably most important are:

(a) The polarization is significantly different from zero at most points.

(b) The sign of the polarization is consistently negative. In particular, there is no indication that the polarization passes through zero in the range studied.

(c) At a given value of $u$, the polarization does not seem to vary much with energy $t$.

We note that, according to conventional Reggepole analysis [5], the pion trajectory, which is supposed to play an important part in determining the cross section for the np charge-exchange reaction, cannot contribute to $P \mathrm{~d} \sigma / \mathrm{d} \Omega \dagger \dagger$. Of the other trajectories which can contribute to the polarization for this reaction, the leading ones are the $\rho$ and $A_{2}$. If only these two trajectories contribute to the polarization, and if they are exchange-degenerate in the most restricted sense (i.e., that both their trajectories coincide and their residue functions are equal), there should be no polarization since the amplitudes would then be relatively real. For $-u<0.3$, our data seem to agree with a prediction by Arnold and Logan [6] who used a weaker form of exchange degeneracy. Their model, however, predicts that $P(u)$ will pass through zero at about $u=-0.5$. We see no sign of this zero-crossing。

We thank Dr. Herbert Steiner for his help in the design of the experiment, and Gary LeBonte and Stephen Wilson for their help with the testing of the spark chambers. Finally we are grateful to the Bevatron operating crew for their excellent support.

$\dagger$ Inspection of fig. 2 shows that $3-4-\mathrm{GeV} / \mathrm{c}$ and $5-$ $5.5-\mathrm{GeV} / c$ data up to $-u=03$ lie systematically below the empirical curve $P=-0.5 \sqrt{-u} / m_{p}$, while the $4-5-\mathrm{GeV} / c$ data in this region of $u$ lie above it. however, it is not yet clear to us, given the size of our errors, whether this is evidence of significant energy dependence

† In the notation of ref. [5], the polarization is given by $P \mathrm{~d} \sigma / \mathrm{d} \Omega=\operatorname{Im} \phi_{5}\left(\phi_{1}+\phi_{2}+\phi_{3}-\phi_{4}\right)^{*}$. For a pion Regge pole exchanged in the $u$ channel the only nonvanishing helicity amplitudes are $\phi_{2}^{\pi}$ and $\phi_{3}^{\pi}$. 'These amplitudes satisfy the condition $\phi_{2}^{\pi}=-\phi_{3}^{\pi}$ and hence give zero contribution to $P \mathrm{~d} \sigma / \mathrm{d} \Omega$, We thank G. L. Kane for calling this fact to our attention. 


\section{References}

1. R.E.Mischke, P.F Shepard and T J.Devlın. Phys Rev. Letters 23 (1969) 542,

G Manning A.G Parham. J D Jafar H.B.van der Raay. D. H. Reading. D. G. Ryan, B. D. Jones, J. Malos and N.H. Lipman, Nuovo Cimento 41A (1966). 167 ,

J.L.Friedes H.Palevsky, R.L.Stearns and R.J. Sutter. Phys. Rev. Letters 15 (1965) 38.

2. For example K Huang and I J.Muzinich, Phys, Rev. 164 (1967) 1726 ,

F.Arab and J.W.Dash, Phys. Rev. 163 (1967) 1603.

3. F.Henyee, G. L. Kane. Jon Pumplin and M H Ross, Phvs. Rev. 182 (1969) 1579 ,
A. B. Kasdalov and B.M.Karnakov. Phys. Letters $29 \mathrm{~B}$ (1969) 372 .

4. N. Byers, Phys. Rev. 156 (1967) 1703 and R.J.N. Phillips, Phys. Letters 4 (1963) 19 are examples of this approach.

5. I.J.Muzinich, Phys. Rev. 130 (1963) 1571.

6. R.C.Arnold and R.K. Logan, Phys. Rev. 177 (1969) 2318. Note that the sign convention for $P(u)$ in their paper is the opposite of ours. They define the normal to the scattering plane as the vector product of the incident neutron and outgoing proton momenta.

ERRATA

Absolute bounds on cross-sections at all energies and without unknown constants. F.J. Ynduráin, Phys. Letters 31B (1970) 368.

The normalization of $a_{2}^{l}$ used is inconsistent. namely, if we keep the definition $a_{n}^{t}=$ $\lim _{q_{t}^{2} \rightarrow 0} f_{2}(t) / q_{t}^{2}$, eq.(6) has to be substituted by

$$
a_{2}^{t}=\frac{16}{15 \pi \mu} \int_{4 \mu}^{\infty} \mathrm{d} s s^{-3} A_{s}\left(s, t=4 \mu^{2}\right) .
$$

The rest of the article is then corrected by substituting everywhere $a_{2}^{t}$ by $3 \pi a_{2}^{t} / 128$. In particular, the numerical coefficient in the third term of the right hand side of eq. (2), $1280 \pi$, has to be substituted by $30 \pi^{2}$.

The estimate does not give. as it stands, the Froissart bound for large $s$, but twice it. To get the Froissart bound, averages of the type $s^{-m-1} \int^{s} \mathrm{~d} s^{\prime} s^{\prime} m_{\sigma_{\text {tot }}} \cdot m$ large, have to be used. Alternatively, one can follow Common's method (A. K. Common, CERN preprint, to be published).
Theory of $\rho^{0}-\omega$ interference in $\pi^{+} \pi^{-}$production, A. S. Goldhaber, G. C. Fox, C.Quigg, Phys. Letters 30B (1969) 249.

In eq. (3), $\frac{1}{m_{\omega}-m-\frac{1}{2} \mathrm{i} \Gamma_{\omega}}$ should read:

$$
\frac{\delta}{m_{\omega}-m-\frac{1}{2} \mathrm{i} \Gamma_{\omega}} \text {. }
$$

$\mathrm{SU}(2) \times \mathrm{SU}(2)$ breaking and the Cabibbo angle, R. J. Oakes, Phys. Letters 29B (1969) 683.

Footnote 4 should include the work of R. Gatto, F. Sartori and M. Tonin, Phys. Letters 28B (1968) 128, where it has also been suggested that $\mathrm{SU}(2) \times \mathrm{SU}(2)$ symmetry breaking and the Cabibbo angle may share a common origin. I am grateful to Professor Gatto for calling my attention to this and regret the oversight. 